\title{
Warped symmetries of the Kerr black hole
}

\author{
Ankit Aggarwal, ${ }^{a, b}$ Alejandra Castro $^{b}$ and Stéphane Detournay ${ }^{a}$ \\ ${ }^{a}$ Physique Mathematique des Interactions Fondamentales, Universite Libre de Bruxelles, \\ Campus Plaine - CP 231, 1050 Bruxelles, Belgium \\ ${ }^{b}$ Institute for Theoretical Physics Amsterdam and Delta Institute for Theoretical Physics, \\ University of Amsterdam, Science Park 904, 1098 XH Amsterdam, The Netherlands \\ E-mail: ankit.aggarwal@ulb.be, a.castro@uva.nl, sdetourn@ulb.ac.be
}

ABSTRACT: We propose a set of diffeomorphism that act non-trivially near the horizon of the Kerr black hole. We follow the recent developments of Haco-Hawking-Perry-Strominger to quantify this phase space, with the most substantial difference being our choice of vectors fields. Our gravitational charges are organized into a Virasoro-Kac-Moody algebra with non-trivial central extensions. We interpret this algebra as arising from a warped conformal field theory. Using the data we can infer from this warped CFT description, we capture the thermodynamic properties of the Kerr black hole.

Keywords: AdS-CFT Correspondence, Black Holes, Conformal Field Theory, SpaceTime Symmetries

ARXIV EPRINT: 1909.03137 


\section{Contents}

1 Introduction 1

2 Black hole monodromy $\quad 3$

3 Warped symmetries in the Kerr geometry $\quad 6$

$\begin{array}{lll}3.1 \text { Central extensions } & 7\end{array}$

4 Black hole thermodynamics from warped symmetries $\quad 9$

5 Discussion 11

$\begin{array}{ll}\text { A Aspects of warped conformal symmetries } & 13\end{array}$

$\begin{array}{ll}\text { A.1 Modular transformations and canonical entropy } & 14\end{array}$

$\begin{array}{ll}\text { B Cardy growth revisited } & 15\end{array}$

C Hidden warped symmetries of the Klein-Gordon operator 16

\section{Introduction}

One remarkable success of the holographic principle is the link it provides between two dimensional conformal field theories, $\mathrm{CFT}_{2}$, and the microscopic origin of black hole thermodynamics. This first connection was observed in the seminal derivation of the microscopic entropy of five-dimensional extremal BPS black holes in the context of string theory by Strominger and Vafa [1]. Despite the specificity of the construction, it provided a framework to explore the robustness of the result beyond its initial scope. Within supersymmetric theories the agreement has been outstanding, allowing for an exploration of both perturbative and non-perturbative effects in quantum gravity; see [2] for a review on the subject. ${ }^{1}$

Soon after this success, it became clear that the key feature was not supersymmetry, or the specific string theory ingredients in [1], but rather the conformal symmetry in the near horizon region. Conformal invariance is implied by the presence of an AdS factor in the near horizon region, and hence AdS/CFT is responsible for the agreement. For example, many of the above supersymmetric solutions contain an $\mathrm{AdS}_{3}$ factor, and it was soon evident that part of the above success relied on $\mathrm{AdS}_{3} / \mathrm{CFT}_{2}$. Among other properties, the entropy of these black holes agrees precisely with the Cardy's growth of states for a $\mathrm{CFT}_{2}[4,5]$. These developments complemented perfectly the derivation of Brown and Henneaux [6], where the symmetry algebra of the so-called large diffeomorphisms forms two copies of a

\footnotetext{
${ }^{1}$ And see [3] for a historical account of these developments in the past two decades.
} 
Virasoro algebra. This placed $\mathrm{AdS}_{3}$ gravity, and the black holes within [7, 8], as a lamppost for our understanding of quantum black holes.

The role of $2 \mathrm{~d}$ conformal symmetry might not be restricted to extremal black holes with a decoupled near-horizon $\mathrm{AdS}_{3}$ region, with early indications already present in [911]. One important observation in this direction was to highlight a hidden conformal symmetry of the Klein-Gordon operator on the Kerr background, which is made manifest when considering a "near" region of phase space rather than spacetime [12]. A second observation is the explicit identification of two sets of Virasoro diffeomorphisms acting on the horizon [13]. The central extensions derived there fill an important gap in this program that address the microscopic origin of the entropy of a generic Kerr black hole with mass $M$ and angular momentum $J$. More concretely, the Cardy formula in a $\mathrm{CFT}_{2}$

$$
S_{\text {Cardy }}=\frac{\pi^{2}}{3} c\left(T_{L}+T_{R}\right) .
$$

with left and right-moving temperatures in [12], and central charge $c=12 J$ obtained in [13], reproduces the Bekenstein-Hawking area law of the Kerr black hole.

The question we would like to ask here is whether the arguments used in $[12,13]$ that point at a description of generic Kerr black holes in terms of a $\mathrm{CFT}_{2}$ are unique. In recent years indeed, alternative holographic scenarios have started to emerge in which the geometries no longer exhibit a local $\mathrm{SL}(2, \mathbb{R}) \times \mathrm{SL}(2, \mathbb{R})$ symmetry, and where the dual field theories differ from a traditional $\mathrm{CFT}_{2}$. One such scenarios involves a new type of field theories, called Warped Conformal Field Theories (WCFT) [14, 15]. We will argue that a description of generic Kerr black holes in terms of a WCFT might appear as natural as a $\mathrm{CFT}_{2}$ one and, in particular, that it allows to reproduce the Bekenstein-Hawking entropy along the lines of [13].

WCFTs are two-dimensional non-relativistic field theories, with an $\mathrm{SL}(2, \mathbb{R}) \times \mathrm{U}(1)$ global symmetry that gets extended into an infinite-dimensional Virasoro-Kac-Moody algebra $[14,15]$. Many of their unique field theoretic properties have been uncovered in recent years [16-27]. In a holographic context, their natural counterparts are gravitational theories that admit Warped $\mathrm{AdS}_{3}\left(\mathrm{WAdS}_{3}\right)$ spacetimes as solutions [28-31]. For several instances of $\mathrm{WAdS}_{3}$ one can establish that the asymptotic symmetries form a Virasoro-Kac-Moody algebra, which is an extension of their local $\mathrm{SL}(2, \mathbb{R}) \times \mathrm{U}(1)$ Killing symmetries [32-38], thus precisely matching the defining symmetries of a WCFT. Remarkably, WCFTs exhibit modular properties that allow to derive a Cardy-type formula for the asymptotic degeneracy of states, which is found to match the entropy of the corresponding $\mathrm{WAdS}_{3}$ black holes [15, 39-45], in the spirit of [4]. This is taken as an indication that WCFTs could be relevant to give a microstate description of certain classes of black holes in lower dimensions. In this work, we will investigate whether WCFTs could also play a role to understand higher dimensional black holes, in particular non-extremal Kerr black holes in 4 dimensions. $^{2}$

\footnotetext{
${ }^{2} \mathrm{WAdS}_{3}$ spaces famously appear in the near-horizon geometry of extremal four-dimensional Kerr black holes [46] central to the Kerr/CFT correspondence [47], which makes this possible holographic description worth exploring.
} 
Our paper is organized as follows. We will start in section 2 by reviewing the analytic properties of the Klein-Gordon operator on the Kerr background. This operator has two regular singular points with non-trivial monodromies, located at the inner and outer horizon of the Kerr black hole. As shown in [48], these monodromies give us a natural basis of energy eigenstates that are crucial for the identification of vector fields we will use section 3 and the identification of $T_{L, R}$ in (1.1). Although this choice of basis is equivalent to the one used in [13], the advantage of phrasing this choice in terms of monodromies is that it does not rely on a low frequency limit of scattering amplitudes: it is an exact feature about the analyticity of an eternal black hole.

Section 3 contains our main result: we will propose a set of vector fields, whose algebra fits the symmetries of a WCFT. As in [13], we use Wald-Zoupas formalism to define covariant charges and evaluate the central extensions of the gravitational charges associated to these vectors. We find a non-trivial central extension for the Virasoro commutator and the Kac-Moody current. One interesting feature of our analysis is that the integrals involved in these terms only receive non-trivial contributions from the future horizon.

In section 4 we discuss how one could use the thermodynamic properties of a WCFT to account for the Bekenstein-Hawking entropy. Using the basis of monodromies in section 2 and the central extensions in section 3, we are able to account for the entropy of the Kerr black hole using the asymptotic formula for the growth of states in a WCFT. This is another piece of evidence that advances a different holographic description of the Kerr black hole.

We end our work with a discussion that includes future directions and weaknesses in this program that are worth mentioning. In appendix A we provide some introductory material on WCFT based on $[15,17]$, and in appendix $\mathrm{B}$ we review the derivation of the Cardy formula using the analogous approach as done for WCFT. Finally in appendix C we discuss how warped symmetries could be hidden in the low frequency limit of a scalar wave equation.

\section{Black hole monodromy}

The metric for the Kerr black hole, in Boyer-Lindquist coordinates, is given by

$$
d s^{2}=\frac{\rho}{\Delta} d r^{2}-\frac{\Delta}{\rho}\left(d t-a \sin ^{2} \theta d \phi\right)^{2}+\rho d \theta^{2}+\frac{\sin ^{2} \theta}{\rho}\left(\left(r^{2}+a^{2}\right) d \phi-a d t\right)^{2},
$$

where $a$ is a constant parameter that controls the rotation, and $\rho=r^{2}+a^{2} \cos ^{2} \theta$. The mass of the black hole $M$ enters the metric via

$$
\Delta=r^{2}+a^{2}-2 M r
$$

The inner and outer horizons are located at

$$
r_{ \pm}=M \pm \sqrt{M^{2}-a^{2}}
$$

which corresponds to the zeroes of $\Delta$. The angular momentum of the black hole is $J=M a$. 
As we discuss physical observables in Kerr, one of our agenda points will be to exploit analytic properties of the black hole background. To motivate our later choices, we will review how analyticity, represented by monodromy data, enters in the structure of the Klein-Gordon equation [48, 49]. The starting point is to consider a massless scalar field $\psi$ in the Kerr background, i.e.

$$
\frac{1}{\sqrt{-g}} \partial_{\mu}\left(\sqrt{-g} g^{\mu \nu} \partial_{\nu} \psi\right)=0
$$

Expanding in eigenmodes

$$
\psi(t, r, \theta, \phi)=e^{-i \omega t+i m \phi} R(r) S(\theta),
$$

the Klein-Gordon operator (2.4) becomes separable. The spheroidal equation is

$$
\left[\frac{1}{\sin \theta} \partial_{\theta}\left(\sin \theta \partial_{\theta}\right)-\frac{m^{2}}{\sin ^{2} \theta}+\omega^{2} a^{2} \cos ^{2} \theta\right] S(\theta)=-K_{\ell} S(\theta)
$$

for eigenvalue $K_{\ell}(a \omega)$; to leading order $K_{\ell}(a \omega)=\ell(\ell+1)+O(a \omega)^{2}$. And the radial equation is given by

$$
\begin{aligned}
& {\left[\partial_{r}\left(r-r_{-}\right)\left(r-r_{+}\right) \partial_{r}+\frac{\left(\omega-\Omega_{+} m\right)^{2}}{4 \kappa_{+}^{2}} \frac{\left(r_{+}-r_{-}\right)}{\left(r-r_{+}\right)}\right.} \\
& \left.\quad-\frac{\left(\omega-\Omega_{-} m\right)^{2}}{4 \kappa_{-}^{2}} \frac{\left(r_{+}-r_{-}\right)}{\left(r-r_{-}\right)}+\left(r^{2}+2 M(r+2 M)\right) \omega^{2}\right] R(r)=K_{\ell} R(r),
\end{aligned}
$$

where

$$
\kappa_{ \pm}=\frac{r_{+}-r_{-}}{4 M r_{ \pm}}, \quad \Omega_{ \pm}=\frac{a}{2 M r_{ \pm}},
$$

are the surface gravity and angular velocity evaluated at the inner $\left(r=r_{-}\right)$and outer horizon $\left(r=r_{+}\right)$.

The global properties of solutions to (2.7) are as follows. Equation (2.7) has two regular singular points at $r=r_{+}$and $r=r_{-}$, which means that the solutions to (2.7) have branch cuts at these points. For instance, around $r=r_{+}$we have two linearly independent solutions

$$
R_{+}^{\text {out }}(r)=\left(r-r_{+}\right)^{i \alpha_{+}}\left(1+O\left(r-r_{+}\right)\right), \quad R_{+}^{\text {in }}(r)=\left(r-r_{+}\right)^{-i \alpha_{+}}\left(1+O\left(r-r_{+}\right)\right),
$$

where

$$
\alpha_{+}=\frac{\left(\omega-\Omega_{+} m\right)}{2 \kappa_{+}} .
$$

This is a convergent series expansion for $R(r)$ when $\left|r-r_{+}\right|<\left|r_{-}-r_{+}\right|$. Near the the inner horizon, $r=r_{-}$, we have a similar expansion, where $\alpha_{+}$is replaced by

$$
\alpha_{-}=\frac{\left(\omega-\Omega_{-} m\right)}{2 \kappa_{-}} .
$$

Here $\alpha_{ \pm}$control the analyticity of the solutions: they are parameters that measure the lack of meromorphicity in $R(r)$ as one transports a solution in the complex $r$-plane around one 
of the singular points. Note that the monodromy eigenvalues $\alpha_{ \pm}$in (2.10)-(2.11) are exact statements about the differential equation; it is not an artefact of a low frequency regime or any other limit.

There is also a singular point at $r=\infty$, and the series expansions reads

$$
R_{\infty}^{\text {out }}(r) \sim e^{i \omega r} r^{i \lambda-1}\left(1+O\left(r^{-1}\right)\right), \quad R_{\infty}^{\text {in }}(r) \sim e^{-i \omega r} r^{-i \lambda-1}\left(1+O\left(r^{-1}\right)\right),
$$

with

$$
\lambda=2 M \omega .
$$

Unlike the singular points at $r=r_{ \pm}$, however, the singular point at $r=\infty$ is irregular. This means that the series (2.12) is asymptotic rather than convergent. The series expansion for $R(r)$ appearing in (2.12) is therefore referred to as a formal solution to the wave equation, as opposed a true solution defined by an analytic continuation of the series expansion (2.9) to the whole complex $r$-plane. The true solution around this irregular point has a monodromy, $\alpha_{\text {irr }}$, which can be computed perturbatively in $\omega$ [49-51]. Its leading behaviour is $i \alpha_{\text {irr }}=$ $\ell+O\left(\omega^{2}\right)$ with $\ell$ the spherical harmonic that controls (2.6).

The monodromy parameters $\alpha_{ \pm}$connect in an elegant manner the scattering coefficients to the analytic properties of the geometry, see e.g. [48, 49, 52, 53]. Their use here will be as energy eigenstates that will motivate our choice of vector fields in section 3 , and impact the holographic interpretation of the thermodynamics of Kerr in section 4 . The canonical choice of eigenstates is to consider functions of the eigenvalues $(\omega, m)$ of the operators $\left(i \partial_{t},-i \partial_{\phi}\right)$, as done in (2.5). Following [48], we will consider instead the linear combinations of the monodromies:

$$
\begin{aligned}
& \omega_{L}:=\alpha_{+}-\alpha_{-}, \\
& \omega_{R}:=\alpha_{+}+\alpha_{-} .
\end{aligned}
$$

To the energies $\omega_{R, L}$ we will assign conjugate variables $t^{ \pm}$, with $\left(\omega_{L}, \omega_{R}\right)$ eigenvalues of $\left(i \partial_{t^{-}}, i \partial_{t^{+}}\right)$. Thus,

$$
e^{-i \omega t+i m \phi}=e^{-i \omega_{L} t^{-}-i \omega_{R} t^{+}} .
$$

Using the explicit form of the monodromies for Kerr (2.10)-(2.11), we find that

$$
t^{+}=2 \pi T_{R} \phi, \quad t^{-}=\frac{1}{2 M} t-2 \pi T_{L} \phi,
$$

where $(t, \phi)$ are Boyer-Lindquist coordinates, and

$$
T_{L}=\frac{r_{+}+r_{-}}{4 \pi a}, \quad T_{R}=\frac{r_{+}-r_{-}}{4 \pi a} .
$$

In position space, we will rewrite $(2.14)$

$$
H_{0}=\frac{i}{2 \pi T_{R}} \partial_{\phi}+2 i M \frac{T_{L}}{T_{R}} \partial_{t}, \quad \bar{H}_{0}=-2 i M \partial_{t} .
$$

It is important to note that these vectors are the same as those used to exhibit the hidden conformal symmetry of Kerr [12], and used in [13]. In our subsequent derivations, a difference worth highlighting, is that we are using the monodromy basis to select (2.18). Moreover we will not interpret (2.18) as a basis for $\mathrm{CFT}_{2}$ energy eigenstates; instead we explore an alternative interpretation in terms of a WCFT description. 


\section{Warped symmetries in the Kerr geometry}

In this section we will introduce a the set of diffeomorphisms in the near horizon geometry of the Kerr black hole, and study the linearized charges associated to them. This section follows closely the proposal in [13], where the most significant deviation comes from our choice of vector fields.

As in $[12,13]$ it is convenient to adapt "conformal" coordinates $\left(w^{ \pm}, y\right)$ defined in terms of $(t, r, \phi)$ by

$$
\begin{aligned}
w^{+} & =\sqrt{\frac{r-r_{+}}{r-r_{-}}} e^{2 \pi T_{R} \phi}, \\
w^{-} & =\sqrt{\frac{r-r_{+}}{r-r_{-}}} e^{2 \pi T_{L} \phi-\frac{t}{2 M}}, \\
y & =\sqrt{\frac{r_{+}-r_{-}}{r-r_{-}}} e^{\pi\left(T_{L}+T_{R}\right) \phi-\frac{t}{4 M}} .
\end{aligned}
$$

A motivation to introduce this coordinate system, is that the local vector fields in (2.18) are now independent of the Kerr black hole parameters; and in particular

$$
H_{0}=i\left(w^{+} \partial_{+}+\frac{1}{2} y \partial_{y}\right), \quad \bar{H}_{0}=i\left(w^{-} \partial_{-}+\frac{1}{2} y \partial_{y}\right) .
$$

An important feature is that under azimuthal identification $\phi \sim \phi+2 \pi$ one finds

$$
w^{+} \sim e^{4 \pi^{2} T_{R}} w^{+}, w^{-} \sim e^{4 \pi^{2} T_{L}} w^{-}, y \sim e^{2 \pi^{2}\left(T_{R}+T_{L}\right)} y .
$$

With the intention of promoting warped symmetries as a holographic description of the Kerr black hole, we will introduce a set of suitably chosen vector fields. These are

$$
\begin{aligned}
& \zeta(\epsilon)=\epsilon\left(w^{+}\right) \partial_{+}+\frac{1}{2} \partial_{+} \epsilon\left(w^{+}\right) y \partial_{y}, \\
& p(\hat{\epsilon})=\hat{\epsilon}\left(w^{+}\right)\left(w^{-} \partial_{-}+\frac{y}{2} \partial_{y}\right),
\end{aligned}
$$

where $\epsilon$ and $\hat{\epsilon}$ are arbitrary functions of $w^{+}$. We restrict these functions such that the vector fields (3.4) are periodic under (3.3); a Fourier decomposition achieving this is

$$
\epsilon_{n}=2 \pi T_{R}\left(w^{+}\right)^{1+\frac{i n}{2 \pi T_{R}}}, \quad \hat{\epsilon}_{n^{\prime}}=\left(w^{+}\right)^{\frac{i n^{\prime}}{2 \pi T_{R}}},
$$

with $n, n^{\prime} \in \mathbb{Z}$, and we define $\zeta_{n} \equiv \zeta\left(\epsilon_{n}\right)$ and $p_{n} \equiv p\left(\hat{\epsilon}_{n}\right)$. With this choice, the Lie bracket of the above vector fields reads

$$
\begin{aligned}
i\left[\zeta_{m}, \zeta_{n}\right] & =(m-n) \zeta_{m+n}, \\
i\left[\zeta_{m}, p_{n}\right] & =n p_{m+n}, \\
i\left[p_{m}, p_{n}\right] & =0
\end{aligned}
$$

which is a Virasoro-KacMoody (VKM) algebra without any central extension. In comparison to the vector field in (3.2), we have

$$
\zeta\left(\epsilon_{0}\right)=-i 2 \pi T_{R} H_{0}, \quad p\left(\hat{\epsilon}_{0}\right)=i \bar{H}_{0},
$$

i.e. our zero modes coincide with the monodromy basis. 


\subsection{Central extensions}

The subsequent analysis will be done using conformal coordinates, and for that purpose it is useful to record some of their properties. The past horizon, which in the Boyer Lindquist systems is at $r=r_{+}, t \in(-\infty, 0)$, maps to $w^{+}=0$; the future horizon, located at $r=r_{+}, t \in(0, \infty)$, maps to $w^{-}=0$. The bifurcation surface $\Sigma_{\text {bif }}$ is therefore at $w^{+}=w^{-}=0$, and around this surface the metric (2.1) becomes

$$
\begin{aligned}
d s^{2}= & \frac{4 \rho_{+}^{2}}{y^{2}} d w^{+} d w^{-}+\frac{16 J^{2} \sin ^{2} \theta}{y^{2} \rho_{+}^{2}} d y^{2}+\rho_{+}^{2} d \theta^{2}-\frac{2 w^{+}(8 \pi J)^{2} T_{R}\left(T_{R}+T_{L}\right)}{y^{3} \rho_{+}^{2}} d w^{-} d y \\
& +\frac{8 w^{-}}{y^{3} \rho_{+}^{2}}\left(-(4 \pi J)^{2} T_{L}\left(T_{R}+T_{L}\right)+\left(4 J^{2}+4 \pi J a^{2}\left(T_{R}+T_{L}\right)+a^{2} \rho_{+}^{2}\right) \sin ^{2} \theta\right) d w^{+} d y \\
& +\cdots
\end{aligned}
$$

where corrections are at least second order in $\left(w^{+}, w^{-}\right)$.

We can associate to the vector fields (3.4) covariant charges; these charges implement the symmetries on a phase space via the Dirac bracket. The linearized charges we will be studying are

$$
\delta \mathcal{Q}=\delta \mathcal{Q}_{\mathrm{IW}}+\delta \mathcal{Q}_{\mathrm{WZ}} .
$$

The first term is the Iyer-Wald charge, which reads

$$
\delta \mathcal{Q}_{\mathrm{IW}}(\chi, h ; g)=\frac{1}{16 \pi} \int_{\partial \Sigma} \star F_{\mathrm{IW}} .
$$

The surface of integration is the bifurcation surface, $\partial \Sigma=\Sigma_{\text {bif }}$. The input in this definition includes a vector field $\chi$, which we will take to be (3.4), a metric perturbation $h_{\mu \nu}$, and the background metric $g_{\mu \nu}$ in (3.8). The integrand is given by

$$
\left(F_{\mathrm{IW}}\right)_{\mu \nu}=\frac{1}{2} \nabla_{\mu} \chi_{\nu} h+\nabla_{\mu} h_{\nu}^{\lambda} \chi_{\lambda}+\nabla_{\lambda} \chi_{\mu} h_{\nu}^{\lambda}-\nabla_{\mu} h \chi_{\nu}-(\mu \leftrightarrow \nu) .
$$

The second term in (3.9) is the Wald-Zoupas counterterm [54], introduced in [13] to comply to some consistency conditions which we will highlight below. Its definition is

$$
\delta \mathcal{Q}_{\mathrm{WZ}}(\chi, h ; g)=\frac{1}{16 \pi} \int_{\partial \Sigma} \iota_{\chi}(\star X), \quad X=2 h_{\mu}{ }^{\nu} \Omega_{\nu} \mathrm{d} x^{\mu},
$$

where $\Omega_{\mu}$ is defined as

$$
\Omega_{\mu}=q_{\mu}^{\lambda} n^{\nu} \nabla_{\lambda} l_{\nu}
$$

The vectors $n^{\mu}$ and $l^{\mu}$ are null, normal to $\Sigma_{\text {bif }}$, and $n \cdot l=-1 ; q_{\mu \nu}=g_{\mu \nu}+n_{\mu} l_{\nu}+n_{\nu} l_{\mu}$ is the induced metric on $\Sigma_{\text {bif }}$. In addition we demand that $n^{\mu}$ and $l^{\mu}$ are single valued under (3.3), which fixes them up to a rescaling.

We are interested in quantifying if the algebra of charges associated to (3.6) admits a central extension. More explicitly, we will define

$$
\delta L_{n} \equiv \delta \mathcal{Q}\left(\zeta_{n}, h ; g\right), \quad \delta P_{n} \equiv \delta \mathcal{Q}\left(p_{n}, h ; g\right),
$$


for the vectors in (3.4) and (3.5). Assuming that these charges are integrable and that the Dirac bracket is well defined, there are 3 possible central extensions. For the Virasoro sector we have

$$
\left[L_{n}, L_{m}\right]=(m-n) L_{m+n}+K_{m, n}, \quad K_{m, n}=\delta \mathcal{Q}\left(\zeta_{n}, \mathcal{L}_{\zeta_{m}} g ; g\right),
$$

and the Kac-Moody generators

$$
\left[P_{n}, P_{m}\right]=k_{m, n}, \quad k_{m, n}=\delta \mathcal{Q}\left(p_{n}, \mathcal{L}_{p_{m}} g ; g\right) .
$$

And finally there is as well the possibility of a mixed central term

$$
\left[L_{n}, P_{m}\right]=m P_{n+m}+\mathfrak{K}_{m, n}, \quad \mathfrak{K}_{m, n}=\delta \mathcal{Q}\left(\zeta_{n}, \mathcal{L}_{p_{m}} g ; g\right) .
$$

The evaluation of these central terms involves some subtleties which we now turn to.

As it was observed in [13], the leading singularities in the integrand of $\delta \mathcal{Q}$ near $\Sigma_{\text {bif }}$ are at most simple poles. For example, in evaluating $K_{m, n}$ there is a non-zero contribution from the $F_{\text {IW }}^{-y}$ component, i.e., from the constant $y$ cross-section of the future horizon close to the bifurcation surface. This term contains a simple pole in $w^{+}$, and the relevant integral to evaluate is

$$
\int_{w_{0}^{+}}^{w_{0}^{+} e^{4 \pi^{2} T_{R}}} \frac{d w^{+}}{w^{+}}=4 \pi^{2} T_{R} .
$$

The limits of $w^{+}$are governed by the identification $w^{+} \sim w^{+} e^{4 \pi^{2} T_{R}}$, and $w_{0}^{+}$is a reference point near $w^{+} \rightarrow 0$. The Wald-Zoupas term in (3.12) has the same type of singular behaviour. The evaluation of $K_{m, n}$ therefore receives contributions from

$$
\begin{gathered}
\delta \mathcal{Q}_{\mathrm{IW}}\left(\zeta_{n}, \mathcal{L}_{\zeta_{m}} g ; g\right)=2 J \frac{T_{R}}{T_{L}+T_{R}}\left(\left(-1+\frac{M^{2}}{a^{2}}\right) m+m^{3}\right) \delta_{n,-m}, \\
\delta \mathcal{Q}_{\mathrm{WZ}}\left(\zeta_{n}, \mathcal{L}_{\zeta_{m}} g ; g\right)=J \frac{T_{L}-T_{R}}{T_{L}+T_{R}}\left(\left(-1+\frac{M^{2}}{a^{2}}\right) m+m^{3}\right) \delta_{n,-m},
\end{gathered}
$$

where we used (3.18) to evaluate the integral over $w^{+}$. The linear term in $m$ can be reabsorbed in the zero mode of the generators, and will be ignored in the following. Adding up the $m^{3}$ contributions gives

$$
K_{m, n}=J m^{3} \delta_{n,-m}
$$

which reproduces the right-movers in [13]. As noted there, individually the terms in (3.19) are problematic since they depend on the mass of the black hole; removing this dependence is the main motivation to introduce the Wald-Zoupas counterterm.

For the two remaining central extensions, the steps are exactly the same: the only nonzero contribution in the Iyer-Wald term (3.10) and Wald-Zoupas counterterm in (3.12) comes from the constant $y$ cross-section of the future horizon close to the bifurcation surface. Thus, we are left to evaluate an integral over $\theta$ and $w^{+}$, where the integral over $w^{+}$is of the form (3.18). This is an important difference relative to [13]: all of our central extension have their pole in the future horizon. We will comment more on this feature in the discussion. 
Carrying out the appropriate integrals, the central extension $k_{m, n}$ in (3.16) receives the two non-trivial contributions which are

$$
\begin{aligned}
\delta \mathcal{Q}_{\mathrm{IW}}\left(p_{n}, \mathcal{L}_{p_{m}} g ; g\right) & =-2 J \frac{T_{L}}{T_{L}+T_{R}} m \delta_{n,-m}, \\
\delta \mathcal{Q}_{\mathrm{WZ}}\left(p_{n}, \mathcal{L}_{p_{m}} g ; g\right) & =J \frac{T_{L}-T_{R}}{T_{L}+T_{R}} m \delta_{n,-m},
\end{aligned}
$$

where these contribution are due to the integrals over the future horizon and we used (3.18). And as in (3.20), the addition of these terms gives a mass independent result; we find

$$
k_{m, n}=-J m \delta_{n,-m}
$$

Finally, our proposed set of vector fields could also allow for a mixed central extension $\mathfrak{K}_{m, n}$. In this case have

$$
\begin{gathered}
\delta \mathcal{Q}_{\mathrm{IW}}\left(\zeta_{n}, \mathcal{L}_{p_{m}} g ; g\right)=i J \frac{T_{R}-T_{L}}{T_{L}+T_{R}}\left(m \frac{i \sqrt{M^{2}-a^{2}}}{a}+m^{2}\right) \delta_{n,-m}, \\
\delta \mathcal{Q}_{\mathrm{WZ}}\left(\zeta_{n}, \mathcal{L}_{p_{m}} g ; g\right)=-i J \frac{T_{R}-T_{L}}{T_{L}+T_{R}}\left(m \frac{i \sqrt{M^{2}-a^{2}}}{a}+m^{2}\right) \delta_{n,-m},
\end{gathered}
$$

which adds to zero, and hence $\mathfrak{K}_{m, n}=0$. It is interesting to note that Wald-Zoupas counterterm here served the additional purpose of eliminating the mixed central extension for our algebra.

Gathering all the results from evaluating the central extensions, the algebra of covariant charges (3.14) associated to the diffeomeorphisms (3.4)-(3.5) seems to obey ${ }^{3}$

$$
\begin{aligned}
{\left[L_{n}, L_{m}\right] } & =(m-n) L_{m+n}+\frac{c}{12} m^{3} \delta_{n,-m}, \\
{\left[L_{n}, P_{m}\right] } & =m P_{n+m} \\
{\left[P_{n}, P_{m}\right] } & =k \frac{m}{2} \delta_{n,-m}
\end{aligned}
$$

with

$$
c=12 J, \quad k=-2 J .
$$

This derivation supports the proposal that the Kerr black hole could be described holographically in terms of a WCFT. The non-trivial central extensions for the VirasoroKacMoody algebra depend on the angular momentum of the black hole.

\section{Black hole thermodynamics from warped symmetries}

In this section we will show how the above information can be used to interpret the entropy of the Kerr black hole

$$
S_{\mathrm{BH}}=\frac{A_{H}}{4}=2 \pi\left(M^{2}+\sqrt{M^{4}-J^{2}}\right)
$$

as the entropy of a thermal state in a WCFT.

\footnotetext{
${ }^{3}$ We emphasise that at this stage we have not evaluated the charges explicitly; we are assuming that they obey suitable Dirac brackets.
} 
The change of coordinates (3.1) can be rewritten, at fixed $r \neq r_{+}$, and up to an $r$-dependent rescaling as

$$
w^{ \pm}=e^{ \pm t^{ \pm}}
$$

with $t^{ \pm}$precisely the coordinates in the monodromy basis (2.16). Since $\phi$ is $2 \pi$-periodic, the corresponding identifications on $t^{ \pm}$make (4.2) the well-known relation between Minkowski $\left(w^{ \pm}\right)$and Rindler $\left(t^{ \pm}\right)$coordinates. In the Minkowski vacuum, observers at a fixed position in Rindler coordinates will detect a thermal radiation.

Now, the orginal Kerr coordinates are subject to both spatial and thermal identifications, respectively

$$
(t, \phi) \sim(t, \phi+2 \pi) \sim(t+i \beta, \phi+\theta),
$$

with $\beta=\frac{2 \pi}{\kappa_{+}}$the inverse Hawking temperature, and $\theta=i \beta \Omega_{+}$the angular potential as defined in (2.8). In terms of (2.16), these identifications read as

$$
\left(t^{+}, t^{-}\right) \sim\left(t^{+}+4 \pi^{2} T_{R}, t^{-}-4 \pi^{2} T_{L}\right) \sim\left(t^{+}+2 \pi i, t^{-}+2 \pi i\right) .
$$

The monodromy analysis therefore suggests that in the natural field theory coordinates $\left(t^{ \pm}\right)$, thermal and spatial cycles are swapped compared to the original Kerr periodicities. This is the case for both the CFT description (which is reviewed in appendix B) and the WCFT one, which we now turn to.

Consider a WCFT defined on a generic torus with spatial and thermal identifications

$$
\left(t^{+}, t^{-}\right) \sim\left(t^{+}-2 \pi \ell, t^{-}+2 \pi \bar{\ell}\right) \sim\left(t^{+}-2 \pi \tau, t^{-}+2 \pi \bar{\tau}\right) .
$$

The entropy in the canonical ensemble, which we review in appendix A, is given by (A.18) and it reads

$$
S_{\bar{\ell} \mid \ell}(\bar{\tau} \mid \tau)=2 \pi i \frac{z}{\hat{t}} \hat{P}_{0}^{\mathrm{vac}}+\frac{4 \pi i}{\hat{t}} \hat{L}_{0}^{\mathrm{vac}},
$$

with $\hat{P}_{0}^{\mathrm{vac}}, \hat{L}_{0}^{\mathrm{vac}}$ are the vacuum values of the zero modes, and

$$
z=\bar{\tau}-\frac{\bar{\ell} \tau}{\ell}, \quad \hat{t}=\frac{\tau}{\ell}
$$

Comparing (4.4) and (4.5), one obtains

$$
\ell=-2 \pi T_{R}, \quad \bar{\ell}=-2 \pi T_{L}, \quad \tau=-i, \quad \bar{\tau}=i,
$$

and hence the entropy is given by

$$
S=4 \pi^{2} i\left(T_{L}+T_{R}\right) \hat{P}_{0}^{\mathrm{vac}}+8 \pi^{2} T_{R} \hat{L}_{0}^{\mathrm{vac}} .
$$

We point out that here $T_{L}$ and $T_{R}$ are defined via the identifications in (4.4); in a WCFT they do not have an interpretation in terms of left and right moving temperatures.

To compare expression (4.9) to the Bekenstein-Hawking entropy of Kerr in (4.1), one needs to identify $\hat{P}_{0}^{\text {vac }}$ and $\hat{L}_{0}^{\text {vac }}$. First note that these two quantities are not independent [15], and related through:

$$
\hat{L}_{0}^{\mathrm{vac}}=-\frac{c}{24}+\frac{\left(\hat{P}_{0}^{\mathrm{vac}}\right)^{2}}{k}
$$


where $c$ and $k$ are the WCFT central extensions. Furthermore, in a WCFT $\hat{P}_{0}^{\text {vac is not }}$ fixed by the symmetries alone [15], unlike what happens in unitary $2 \mathrm{~d}$ CFTs. To fix this ambiguity we will borrow from other instances of WCFT in holography. A consistent pattern in holographic setups, such as [15, 32-34, 39, 55-57], is

$$
\hat{L}_{0}^{\mathrm{vac}}=0,
$$

where the common thread is the absence of a gravitational anomaly in the bulk theory, such as a gravitational Chern-Simons term. ${ }^{4}$ Note that this pattern renders an imaginary value for $\hat{P}_{0}^{\text {vac }}$ in holography. It is natural to assume that (4.11) holds for our circumstances too! Using the values of the central extensions in (3.25) and (4.11) in (4.10) gives

$$
\left(\hat{P}_{0}^{\mathrm{vac}}\right)^{2}=-J^{2}
$$

and the entropy (4.9) is simply

$$
S=4 \pi^{2}|J|\left(T_{L}+T_{R}\right)
$$

Plugging (2.17), and (2.3), one obtains that (4.9) exactly agrees with (4.1).

\section{Discussion}

We found evidence that a WCFT could be a suitable holographic description of the Kerr black hole. Many conceptual pieces are missing in this description and several aspects of this proposal are mysterious to us. In the following we will discuss future directions that could address these issues.

1. One notable aspect of our computation is that the contribution to the central extensions comes only from a component of the future horizon that is near the bifurcation surface at constant $y .{ }^{5}$ This hints at the possibility that these symmetries could be used to understand more dynamical situations, like black holes formed by collapse. However, it still remains to be seen whether the charges also follow a similar pattern. As a first step in this direction, we have tried to compute the charges for the Kerr background. Here, we found that the charges receive a finite contribution from the future horizon. However, the other two surfaces cause problems. The contributions from these surfaces are ill-defined unless $\delta r_{+}=0$, i.e., the horizon radius is kept fixed in the phase space. This is not what we have been using in the central extension computations, where we fixed the angular momentum $J$. It is important to clarify this issue and better understand the phase space.

To get some insight in this matter, we did similar computations in three dimensions for the BTZ black hole in Einstein gravity (and TMG) where the phase space is better understood. We used the natural analogs of the conformal metric (3.1) and vector

\footnotetext{
${ }^{4}$ Gravitational anomalies in local WCFTs have also been investigated in [23].

${ }^{5}$ In contrast, the central extensions in [13] have contributions from the future horizon for their right moving vector fields, and past horizon for the left movers.
} 
fields (3.4) adapted to the 3D black hole. After a computation almost identical to the one for Kerr, ${ }^{6}$ we get the CSS central extensions [58], and their TMG counterpart. For the charges of the BTZ background, we again find that the future horizon gives finite contributions. The other surfaces contribute pathologically unless $\delta r_{+}=0$, as for the Kerr background. A similar study done for Warped BTZ in TMG also reproduces the correct central extensions. All these studies in $3 \mathrm{~d}$ reinforce our confidence for the Kerr computation presented in this work. However, the important issues regarding the charges and phase space for generic Kerr still remain, and are left for future investigations.

In this regard, it would be interesting to discuss potential ambiguities in the definition of the charges. As proposed in [13] and as we discussed in this paper, a Wald-Zoupas boundary counterterm had to be added to make the central charge state-independent. It is not known whether the term used here is unique, or if there exists an alternative term that would make both the central charges state-independent and the nearhorizon charges well-defined for example. It would be clearly desirable to find a first principle derivation fixing this boundary term, for instance by requiring to have a well-defined variational problem.

2. Our work illustrates that there is ambiguity on defining horizon symmetries for nonextremal black holes. Earlier related works by Carlip studying horizon symmetries for generic black holes exhibit the same ambiguity: depending on the choice of boundary conditions at the horizon, the symmetries can be either conformal [59-62] or $\mathrm{BMS}_{3}$ [63], and both seem able to account for the black hole entropy. It is also worth mentioning that various proposals for boundary conditions at non-extremal horizons in three and four dimensions have appeared in recent years [41, 64-72]. The ambiguity in the choice of boundary conditions is reminiscent of the archetypical $\mathrm{AdS}_{3}$ gravity setting: besides the Brown-Henneaux boundary conditions, a handful of alternative boundary conditions have appeared in recent years with symmetries differing from those of a pure $\mathrm{CFT}_{2}[41,58,64,65,73-76]$. In the case at hand, it would be interesting to investigate the existence of boundary conditions with asymptotic symmetries given by (3.4).

3. Our derivation of the Bekenstein-Hawking entropy via (4.9) has two important weaknesses. First we have not derived the vacuum value (4.11), nor identified the corresponding geometry. This is tied to our lack of knowledge of the classical phase space, which is a persistent shortcoming of works related to the Kerr/CFT correspondence.

Second, the derivation of (4.6), as outlined in appendix A.1, is strictly valid in the high temperature regime. In particular it assumes that $c$ and $k$ are fixed, and $T_{L, R} \gg 1$. In the gravitational side, the temperatures are constrained via $T_{L}^{2}-T_{R}^{2}=$

\footnotetext{
${ }^{6}$ For BTZ we only need to evaluate the Iyer-Wald charge in (3.10) to reproduce, for example, the Brown-Henneaux central charge [6]. The Wald-Zoupas counterterm (3.12) gives a finite contribution to the central extension that depends on the charges of BTZ. The comments in this paragraph are based on using only (3.10) in the definition of covariant charges.
} 
$1 / 4 \pi^{2}$, which in particular allows them to have small values as the black hole reaches extremality. This is somewhat similar to the non-trivial conditions one needs to impose on a $\mathrm{CFT}_{2}$ such that entropy of the BTZ black hole is fully captured [77]. Understanding the conditions on WCFTs that accommodate for the thermodynamic regime of the black hole remains an open problem worth exploring.

\section{Acknowledgments}

We thank Geoffrey Compère, Laura Donnay, Tom Hartman, Diego Hofman, Andrea Puhm, Andrew Strominger and Céline Zwikel for useful discussions. We also thank the hospitality of the Erwin Schrödinger Institute (ESI) in Vienna in March 2019 during the program 'Higher Spins and Holography', where part of this work was completed. AC is supported by Nederlandse Organisatie voor Wetenschappelijk Onderzoek (NWO) via a Vidi grant, and by the Delta ITP consortium, a program of the NWO that is funded by the Dutch Ministry of Education, Culture and Science (OCW). AA and SD are supported in part by the ARC grant "Holography, Gauge Theories and Quantum Gravity Building models of quantum black holes", by IISN - Belgium (convention 4.4503.15) and benefited from the support of the Solvay Family. SD is a Research Associate of the Fonds de la Recherche Scientifique F.R.S.-FNRS (Belgium).

\section{A Aspects of warped conformal symmetries}

In this appendix we record some basic features of warped conformal symmetries, based on $[15,17]$. We will focus on the symmetries, conserved charges and modular properties associated to a warped conformal field theory.

Consider a $(1+1)$ dimensional theory defined on a plane which we describe in terms of two coordinates $(u, v)$. On this plane, we denote as $T(u)$ the operator that generates infinitesimal coordinate transformations in $u$ and $P(u)$ the operator that generates $u$ dependent infinitesimal translations in $v$. The corresponding coordinate transformations are

$$
u \rightarrow u=f\left(u^{\prime}\right), \quad v \rightarrow v=v^{\prime}+g\left(u^{\prime}\right) .
$$

In the quantum theory, $T(u)$ is a right moving energy momentum tensor and $P(u)$ as a right moving $\mathrm{U}(1)$ Kac-Moody current. The associated charges are

$$
L_{n}=-\frac{i}{2 \pi} \int d u \zeta_{n}(u) T(u), \quad P_{n}=-\frac{1}{2 \pi} \int d u \chi_{n}(u) P(u),
$$

where we choose the test functions as $\zeta_{n}=u^{n+1}$ and $\chi_{n}=u^{n}$. In terms of the plane charges $\left(L_{n}, P_{n}\right)$ the commutation relations are

$$
\begin{aligned}
& {\left[L_{n}, L_{n^{\prime}}\right]=\left(n-n^{\prime}\right) L_{n+n^{\prime}}+\frac{c}{12} n\left(n^{2}-1\right) \delta_{n,-n^{\prime}},} \\
& {\left[L_{n}, P_{n^{\prime}}\right]=-n^{\prime} P_{n^{\prime}+n},} \\
& {\left[P_{n}, P_{n^{\prime}}\right]=k \frac{n}{2} \delta_{n,-n^{\prime}},}
\end{aligned}
$$


which is a Virasoro-Kac-Moody algebra with central charge $c$ and level $k$. The finite transformation properties of the operators are

$$
\begin{aligned}
P^{\prime}\left(u^{\prime}\right) & =\frac{\partial u}{\partial u^{\prime}}\left(P(u)+\frac{k}{2} \frac{\partial v^{\prime}}{\partial u}\right), \\
T^{\prime}\left(u^{\prime}\right) & =\left(\frac{\partial u}{\partial u^{\prime}}\right)^{2}\left(T(u)-\frac{c}{12}\left\{u^{\prime}, u\right\}\right)+\frac{\partial u}{\partial u^{\prime}} \frac{\partial v}{\partial u^{\prime}} P(u)-\frac{k}{4}\left(\frac{\partial v}{\partial u^{\prime}}\right)^{2},
\end{aligned}
$$

where

$$
\left\{u^{\prime}, u\right\}=\frac{\frac{\partial^{3} u^{\prime}}{\partial u^{3}}}{\frac{\partial u^{\prime}}{\partial u}}-\frac{3}{2}\left(\frac{\frac{\partial^{2} u^{\prime}}{\partial u^{2}}}{\frac{\partial u^{\prime}}{\partial u}}\right)^{2} .
$$

\section{A.1 Modular transformations and canonical entropy}

Consider an arbitrary torus defined by identifications

$$
(u, v) \sim(u-2 \pi \ell, v+2 \pi \bar{\ell}) \sim(u-2 \pi \tau, v+2 \pi \bar{\tau}) .
$$

The WCFT partition function on this torus is given by

$$
Z_{\bar{\ell} \mid \ell}(\bar{\tau} \mid \tau)=\operatorname{Tr}_{\bar{\ell} \mid \ell}\left(e^{2 \pi i \bar{\tau} P_{0}} e^{-2 \pi i \tau L_{0}}\right) .
$$

where the label $(\bar{\ell} \mid \ell)$ denotes the choice of torus. $P_{0}$ and $L_{0}$ denote the zero modes of the WCFT generators:

$$
P_{0}=-\frac{1}{2 \pi} \int_{0}^{2 \pi \ell} P(u) d u, \quad L_{0}=-\frac{1}{2 \pi} \int_{0}^{2 \pi \ell} T(u) d u .
$$

One can do the following warped conformal transformation

$$
\hat{u}=\frac{u}{\ell}, \quad \hat{v}=v+\frac{\bar{\ell}}{\ell} u,
$$

which brings the generic torus in (A.6) to a canonical torus, i.e. it sets $\bar{\ell}=0$ and $\ell=1$. This transformation affects also the zero modes, as dictated by (A.4), which allows us to relate the partition function on (A.6) and (A.9) as

$$
Z_{\bar{\ell} \mid \ell}(\bar{\tau} \mid \tau)=e^{\pi i k \bar{\ell}\left(\bar{\tau}-\frac{\bar{\ell} \tau}{2 \ell}\right)} \hat{Z}\left(\bar{\tau}-\frac{\bar{\ell} \tau}{\ell} \mid \frac{\tau}{\ell}\right)
$$

where

$$
\hat{Z}(\hat{\bar{\tau}} \mid \hat{\tau}):=Z_{0 \mid 1}(\hat{\bar{\tau}} \mid \hat{\tau})=\operatorname{Tr}_{0 \mid 1}\left(e^{2 \pi i \hat{\bar{\tau}} \hat{P}_{0}} e^{-2 \pi i \hat{\bar{\tau}} \hat{L}_{0}}\right) .
$$

The statement that a WCFT is invariant under an $S$-transformation implies

$$
Z_{0 \mid 1}(\bar{\tau} \mid \tau)=Z_{\bar{\tau} \mid \tau}(0 \mid-1) .
$$

This allows us to rewrite (A.10) as

$$
\hat{Z}(z \mid t)=e^{\pi i k \frac{z^{2}}{2 t}} \hat{Z}\left(\frac{z}{t} \mid \frac{-1}{t}\right) .
$$


Thus, a generic partition function can be written as

$$
Z_{\bar{\ell} \mid \ell}(\bar{\tau} \mid \tau)=e^{\pi i k \bar{\ell}\left(\bar{\tau}-\frac{\tau \bar{\ell}}{2 \ell}\right)+\pi i k \frac{z^{2}}{2 t}} \hat{Z}\left(\frac{z}{t} \mid \frac{-1}{t}\right)=e^{\pi i k \frac{\ell \bar{\tau}^{2}}{2 \tau}} \hat{Z}\left(\frac{z}{t} \mid \frac{-1}{t}\right),
$$

where

$$
z=\bar{\tau}-\frac{\bar{\ell} \tau}{\ell}, \quad t=\frac{\tau}{\ell}
$$

The entropy is defined by

$$
S=\left(1-\tau \partial_{\tau}-\bar{\tau} \partial_{\bar{\tau}}\right) \log Z
$$

We are interested in extracting the entropy in the high temperature regime, i.e. in the limit $t \rightarrow-i 0$. If $z$ is purely imaginary and the spectrum of $L_{0}$ is bounded below one can use (A.14) to obtain the projected partition function

$$
Z \rightarrow e^{\pi i k \frac{\ell \bar{\tau}^{2}}{2 \tau}} e^{2 \pi i \frac{z}{t} \hat{P}_{0}^{\mathrm{vac}}} e^{\frac{2 \pi i}{t} \hat{L}_{0}^{\mathrm{vac}}} .
$$

In other words, we can approximate the partition function by the vacuum state on the canonical torus. Using (A.17) in (A.16), the entropy in this regime is

$$
S_{\bar{\ell} \mid \ell}(\bar{\tau} \mid \tau)=2 \pi i \frac{z}{t} \hat{P}_{0}^{\mathrm{vac}}+\frac{4 \pi i}{t} \hat{L}_{0}^{\mathrm{vac}}
$$

with

$$
z=\bar{\tau}-\frac{\bar{\ell} \tau}{\ell}, \quad t=\frac{\tau}{\ell}
$$

Here $\hat{P}_{0}^{\mathrm{vac}}, \hat{L}_{0}^{\mathrm{vac}}$ are the vacuum values of the zero modes on the canonical torus.

\section{B Cardy growth revisited}

In this appendix we revisit the derivation of the entropy in the canonical ensemble for a $\mathrm{CFT}_{2}$. The goal is to set the derivation in the same language as done in appendix A.1 for a WCFT.

Consider a $2 \mathrm{~d}$ CFT on a torus with symmetries $u \rightarrow f(u)$ and $v \rightarrow f(v)$ defined by the following identifications:

$$
(u, v) \sim(u-2 \pi \ell, v+2 \pi \bar{\ell}) \sim(u-2 \pi \tau, v+2 \pi \bar{\tau}) .
$$

The partition function is written

$$
Z_{\bar{\ell} \mid \ell}(\bar{\tau} \mid \tau)=\operatorname{Tr}_{\bar{\ell} \mid \ell}\left(e^{2 \pi i \bar{\tau} \bar{L}_{0}} e^{-2 \pi i \tau L_{0}}\right) .
$$

Using the transformation $u^{\prime}=\lambda^{+} u$ and $v^{\prime}=\lambda^{+} v$, one can map the theory on a canonical circle $(\bar{\ell}, \ell)=(1,1)$, which implies

$$
Z_{\bar{\ell} \mid \ell}(\bar{\tau} \mid \tau)=Z_{1 \mid 1}\left(\frac{\bar{\tau}}{\bar{\ell}} \mid \frac{\tau}{\ell}\right)
$$


The modular S-transformation in 2d CFTs is

$$
Z_{1 \mid 1}(\bar{\tau} \mid \tau)=Z_{1 \mid 1}\left(-\frac{1}{\bar{\tau}} \mid-\frac{1}{\tau}\right)
$$

From this, one gets

$$
Z_{\bar{\ell} \mid \ell}(\bar{\tau} \mid \tau)=Z_{1 \mid 1}\left(-\frac{\bar{\ell}}{\bar{\tau}} \mid-\frac{\ell}{\tau}\right) .
$$

At small temperatures, the right hand side can be projected on the vacuum state on the canonical torus, whose charges are denoted by $L_{0}^{\mathrm{vac}}$ and $\bar{L}_{0}^{\mathrm{vac}}$, to obtain

$$
\log Z_{\bar{\ell} \mid \ell}(\bar{\tau} \mid \tau) \approx-2 \pi i \frac{\bar{\ell}}{\bar{\tau}} \bar{L}_{0}^{\mathrm{vac}}+2 \pi i \frac{\ell}{\tau} L_{0}^{\mathrm{vac}}
$$

from which one gets the entropy

$$
S_{\bar{\ell} \mid \ell}(\bar{\tau} \mid \tau) \approx-4 \pi i \frac{\bar{\ell}}{\bar{\tau}} \bar{L}_{0}^{\mathrm{vac}}+4 \pi i \frac{\ell}{\tau} L_{0}^{\mathrm{vac}} .
$$

This is the well known Cardy formula in the canonical ensemble, which we are recasting for a general torus.

For the Kerr black hole, the identifications that define the Euclidean geometry are

$$
(t, \phi) \sim(t, \phi-2 \pi) \sim\left(t+i \beta_{K}, \phi+\theta_{K}\right) .
$$

From (4.4), we identify $u \leftrightarrow t^{+}$and $v \leftrightarrow t^{-}$, and so

$$
\ell=2 \pi T_{R}, \quad \bar{\ell}=2 \pi T_{L}, \quad \tau=-i, \quad \bar{\tau}=i .
$$

Using these values (B.7) together with $L_{0}^{\mathrm{vac}}=\bar{L}_{0}^{\mathrm{vac}}=-\frac{c}{24}$, leads to

$$
S=\frac{\pi^{2}}{3} c\left(T_{L}+T_{R}\right),
$$

which is the Cardy formula reported in (1.1).

\section{Hidden warped symmetries of the Klein-Gordon operator}

In this appendix we will revisit the hidden conformal symmetries of [12]: we will show how a mild modification to the "near region" allows for an additional term in the wave equation. This addition is naturally interpreted in terms of a hidden warped symmetry.

We will define the "near region" as region in phase space where we will impose

$$
r \omega \ll 1, \quad M \omega \ll 1 .
$$

In the regime (C.1), the wave equation (2.7) leads to

$$
\begin{aligned}
{\left[\partial_{r}\left(r-r_{-}\right)\left(r-r_{+}\right) \partial_{r}\right.} & +\frac{\left(\omega-\Omega_{+} m\right)^{2}}{4 \kappa_{+}^{2}} \frac{\left(r_{+}-r_{-}\right)}{\left(r-r_{+}\right)} \\
& \left.-\frac{\left(\omega-\Omega_{-} m\right)^{2}}{4 \kappa_{-}^{2}} \frac{\left(r_{+}-r_{-}\right)}{\left(r-r_{-}\right)}+4 M^{2} \omega^{2}\right] R(r)=K_{\ell} R(r),
\end{aligned}
$$


Relative to the limit in [12], where the term last in the bracket is absent, we are arguing here that the terms

$$
\frac{\left(\omega-\Omega_{ \pm} m\right)^{2}}{4 \kappa_{ \pm}^{2}} \frac{\left(r_{+}-r_{-}\right)}{\left(r-r_{ \pm}\right)}, \quad M^{2} \omega^{2},
$$

are the leading and subleading contributions in frequency space, while $r \omega$ contributions in (2.7) are negligible relative to these terms. Note that $K_{\ell}$ also receives $\omega^{2}$ corrections which should be accounted for too. In terms of analyticity of the wave equation, we are keeping the data of the two regular singular points at $r_{ \pm}$and the constant term in the effective potential; this transforms the irregular singular point at $r \rightarrow \infty$ into a regular singularity.

Although we have one additional term in this regime, we can write the wave equation (C.2) as

$$
\left(\mathcal{H}^{2}-\bar{H}_{0}^{2}\right) R(r)=K_{\ell} R(r),
$$

where $\mathcal{H}^{2}$ is the quadratic Casimir of an $\operatorname{sl}(2, \mathbb{R})$ algebra

$$
\mathcal{H}^{2}=-H_{0}^{2}+\frac{1}{2}\left(H_{1} H_{-1}+H_{-1} H_{1}\right),
$$

and the generators are

$$
\begin{aligned}
H_{1} & =i e^{-2 \pi T_{R} \phi}\left(\Delta^{1 / 2} \partial_{r}+\frac{1}{2 \pi T_{R}} \frac{r-M}{\Delta^{1 / 2}} \partial_{\phi}+\frac{2 T_{L}}{T_{R}} \frac{M r-a^{2}}{\Delta^{1 / 2}} \partial_{t}\right), \\
H_{0} & =\frac{i}{2 \pi T_{R}} \partial_{\phi}+2 i M \frac{T_{L}}{T_{R}} \partial_{t}, \\
H_{-1} & =i e^{2 \pi T_{R} \phi}\left(-\Delta^{1 / 2} \partial_{r}+\frac{1}{2 \pi T_{R}} \frac{r-M}{\Delta^{1 / 2}} \partial_{\phi}+\frac{2 T_{L}}{T_{R}} \frac{M r-a^{2}}{\Delta^{1 / 2}} \partial_{t}\right),
\end{aligned}
$$

The additional generator in (C.4) is just a $u(1)$ generator defined as

$$
\bar{H}_{0}=-2 i M \partial_{t}
$$

which encodes the additional $4 M^{2} \omega^{2}$ term in (C.2).

The interpretation of (C.4) is interesting. For fixed $K_{\ell}$, the solutions can be organized as representations of $s l(2) \times u(1)$, which is compatible with the global isometries of a WCFT. Moreover the wave equations of the form (C.4) can be identified as those that arise from thermal Warped $\mathrm{AdS}_{3}$ geometries; see for example section 5 of [22], where the WAdS/WCFT Green's function are discussed.

Open Access. This article is distributed under the terms of the Creative Commons Attribution License (CC-BY 4.0), which permits any use, distribution and reproduction in any medium, provided the original author(s) and source are credited.

\section{References}

[1] A. Strominger and C. Vafa, Microscopic origin of the Bekenstein-Hawking entropy, Phys. Lett. B 379 (1996) 99 [hep-th/9601029] [INSPIRE]. 
[2] A. Sen, Microscopic and macroscopic entropy of extremal black holes in string theory, Gen. Rel. Grav. 46 (2014) 1711 [arXiv: 1402.0109] [InSPIRE].

[3] S. De Haro, J. van Dongen, M. Visser and J. Butterfield, Conceptual analysis of black hole entropy in string theory, arXiv:1904.03232 [INSPIRE].

[4] A. Strominger, Black hole entropy from near horizon microstates, JHEP 02 (1998) 009 [hep-th/9712251] [INSPIRE].

[5] J.M. Maldacena and A. Strominger, AdS $S_{3}$ black holes and a stringy exclusion principle, JHEP 12 (1998) 005 [hep-th/9804085] [INSPIRE].

[6] J.D. Brown and M. Henneaux, Central charges in the canonical realization of asymptotic symmetries: an example from three-dimensional gravity, Commun. Math. Phys. 104 (1986) 207.

[7] M. Bañados, C. Teitelboim and J. Zanelli, The black hole in three-dimensional space-time, Phys. Rev. Lett. 69 (1992) 1849 [hep-th/9204099] [INSPIRE].

[8] M. Bañados, M. Henneaux, C. Teitelboim and J. Zanelli, Geometry of the $(2+1)$ black hole, Phys. Rev. D 48 (1993) 1506 [Erratum ibid. D 88 (2013) 069902] [gr-qc/9302012] [INSPIRE].

[9] J.M. Maldacena and A. Strominger, Universal low-energy dynamics for rotating black holes, Phys. Rev. D 56 (1997) 4975 [hep-th/9702015] [INSPIRE].

[10] M. Cvetič and F. Larsen, Grey body factors for rotating black holes in four-dimensions, Nucl. Phys. B 506 (1997) 107 [hep-th/9706071] [INSPIRE].

[11] M. Cvetič and F. Larsen, Greybody factors for black holes in four-dimensions: particles with spin, Phys. Rev. D 57 (1998) 6297 [hep-th/9712118] [INSPIRE].

[12] A. Castro, A. Maloney and A. Strominger, Hidden conformal symmetry of the Kerr black hole, Phys. Rev. D 82 (2010) 024008 [arXiv: 1004.0996] [INSPIRE].

[13] S. Haco, S.W. Hawking, M.J. Perry and A. Strominger, Black hole entropy and soft hair, JHEP 12 (2018) 098 [arXiv: 1810.01847] [INSPIRE].

[14] D.M. Hofman and A. Strominger, Chiral scale and conformal invariance in $2 D$ quantum field theory, Phys. Rev. Lett. 107 (2011) 161601 [arXiv:1107.2917] [INSPIRE].

[15] S. Detournay, T. Hartman and D.M. Hofman, Warped conformal field theory, Phys. Rev. D 86 (2012) 124018 [arXiv: 1210.0539] [INSPIRE].

[16] D.M. Hofman and B. Rollier, Warped conformal field theory as lower spin gravity, Nucl. Phys. B 897 (2015) 1 [arXiv: 1411.0672] [INSPIRE].

[17] A. Castro, D.M. Hofman and G. Sárosi, Warped Weyl fermion partition functions, JHEP 11 (2015) 129 [arXiv: 1508.06302] [INSPIRE].

[18] S. Detournay and C. Zwikel, Phase transitions in warped $A d S_{3}$ gravity, JHEP 05 (2015) 074 [arXiv: 1504.00827] [INSPIRE].

[19] A. Castro, D.M. Hofman and N. Iqbal, Entanglement entropy in warped conformal field theories, JHEP 02 (2016) 033 [arXiv:1511.00707] [INSPIRE].

[20] W. Song, Q. Wen and J. Xu, Modifications to holographic entanglement entropy in warped CFT, JHEP 02 (2017) 067 [arXiv:1610.00727] [INSPIRE]. 
[21] W. Song, Q. Wen and J. Xu, Generalized gravitational entropy for warped Anti-de Sitter space, Phys. Rev. Lett. 117 (2016) 011602 [arXiv:1601.02634] [INSPIRE].

[22] W. Song and J. Xu, Correlation functions of warped CFT, JHEP 04 (2018) 067 [arXiv: 1706.07621] [INSPIRE].

[23] K. Jensen, Locality and anomalies in warped conformal field theory, JHEP 12 (2017) 111 [arXiv: 1710.11626] [INSPIRE].

[24] L. Apolo and W. Song, Bootstrapping holographic warped CFTs or: how I learned to stop worrying and tolerate negative norms, JHEP 07 (2018) 112 [arXiv:1804.10525] [INSPIRE].

[25] L. Apolo, S. He, W. Song, J. Xu and J. Zheng, Entanglement and chaos in warped conformal field theories, JHEP 04 (2019) 009 [arXiv:1812.10456] [INSPIRE].

[26] W. Song and J. Xu, Structure constants from modularity in warped CFT, JHEP 10 (2019) 211 [arXiv: 1903.01346] [INSPIRE].

[27] B. Chen, P.-X. Hao and W. Song, Rényi mutual information in holographic warped CFTs, JHEP 10 (2019) 037 [arXiv: 1904.01876] [INSPIRE].

[28] D. Israel, C. Kounnas, D. Orlando and P.M. Petropoulos, Electric/magnetic deformations of $S^{3}$ and $A d S_{3}$ and geometric cosets, Fortsch. Phys. 53 (2005) 73 [hep-th/0405213] [INSPIRE].

[29] M. Rooman and P. Spindel, Godel metric as a squashed Anti-de Sitter geometry, Class. Quant. Grav. 15 (1998) 3241 [gr-qc/9804027] [INSPIRE].

[30] K.A. Moussa, G. Clement and C. Leygnac, The black holes of topologically massive gravity, Class. Quant. Grav. 20 (2003) L277 [gr-qc/0303042] [INSPIRE].

[31] D. Anninos, W. Li, M. Padi, W. Song and A. Strominger, Warped AdS $S_{3}$ black holes, JHEP 03 (2009) 130 [arXiv:0807.3040] [InSPIRE].

[32] G. Compere and S. Detournay, Centrally extended symmetry algebra of asymptotically Gödel spacetimes, JHEP 03 (2007) 098 [hep-th/0701039] [INSPIRE].

[33] G. Compere and S. Detournay, Semi-classical central charge in topologically massive gravity, Class. Quant. Grav. 26 (2009) 012001 [Erratum ibid. 26 (2009) 139801] [arXiv:0808.1911] [INSPIRE].

[34] G. Compere and S. Detournay, Boundary conditions for spacelike and timelike warped $A d S_{3}$ spaces in topologically massive gravity, JHEP 08 (2009) 092 [arXiv:0906.1243] [INSPIRE].

[35] D. Anninos et al., The curious case of null warped space, JHEP 11 (2010) 119 [arXiv: 1005.4072] [INSPIRE].

[36] M. Henneaux, C. Martinez and R. Troncoso, Asymptotically warped Anti-de Sitter spacetimes in topologically massive gravity, Phys. Rev. D 84 (2011) 124016 [arXiv:1108.2841] [INSPIRE].

[37] M. Blagojevic and B. Cvetkovic, Asymptotic structure of topologically massive gravity in spacelike stretched AdS sector, JHEP 09 (2009) 006 [arXiv:0907.0950] [INSPIRE].

[38] D. Anninos, S. de Buyl and S. Detournay, Holography for a de Sitter-esque geometry, JHEP 05 (2011) 003 [arXiv: 1102.3178] [INSPIRE].

[39] L. Donnay and G. Giribet, Holographic entropy of warped-AdS 3 black holes, JHEP 06 (2015) 099 [arXiv: 1504.05640] [INSPIRE]. 
[40] G. Giribet and M. Tsoukalas, Warped-AdS 3 black holes with scalar halo, Phys. Rev. D 92 (2015) 064027 [arXiv: 1506. 05336] [INSPIRE].

[41] H. Afshar, S. Detournay, D. Grumiller and B. Oblak, Near-horizon geometry and warped conformal symmetry, JHEP 03 (2016) 187 [arXiv: 1512.08233] [INSPIRE].

[42] S. Detournay, L.-A. Douxchamps, G.S. Ng and C. Zwikel, Warped AdS $S_{3}$ black holes in higher derivative gravity theories, JHEP 06 (2016) 014 [arXiv:1602.09089] [INSPIRE].

[43] C. Zwikel, BTZ black holes and flat space cosmologies in higher derivative theories, Class. Quant. Grav. 34 (2017) 085003 [arXiv: 1604.02120] [INSPIRE].

[44] M.R. Setare and H. Adami, Asymptotically spacelike warped Anti-de Sitter spacetimes in generalized minimal massive gravity, Class. Quant. Grav. 34 (2017) 125008 [arXiv: 1701.00209] [INSPIRE].

[45] T. Azeyanagi, S. Detournay and M. Riegler, Warped black holes in lower-spin gravity, Phys. Rev. D 99 (2019) 026013 [arXiv: 1801.07263] [INSPIRE].

[46] J.M. Bardeen and G.T. Horowitz, The extreme Kerr throat geometry: a vacuum analog of $A d S_{2} \times S^{2}$, Phys. Rev. D 60 (1999) 104030 [hep-th/9905099] [INSPIRE].

[47] M. Guica, T. Hartman, W. Song and A. Strominger, The Kerr/CFT correspondence, Phys. Rev. D 80 (2009) 124008 [arXiv:0809.4266] [INSPIRE].

[48] A. Castro, J.M. Lapan, A. Maloney and M.J. Rodriguez, Black hole monodromy and conformal field theory, Phys. Rev. D 88 (2013) 044003 [arXiv: 1303.0759] [INSPIRE].

[49] A. Castro, J.M. Lapan, A. Maloney and M.J. Rodriguez, Black hole scattering from monodromy, Class. Quant. Grav. 30 (2013) 165005 [arXiv:1304.3781] [INSPIRE].

[50] S. Mano, H. Suzuki and E. Takasugi, Analytic solutions of the Teukolsky equation and their low frequency expansions, Prog. Theor. Phys. 95 (1996) 1079 [gr-qc/9603020] [InSPIRE].

[51] S. Mano and E. Takasugi, Analytic solutions of the Teukolsky equation and their properties, Prog. Theor. Phys. 97 (1997) 213 [gr-qc/9611014] [InSPIRE].

[52] F. Novaes and B. Carneiro da Cunha, Isomonodromy, Painlevé transcendents and scattering off of black holes, JHEP 07 (2014) 132 [arXiv: 1404.5188] [INSPIRE].

[53] B. Carneiro da Cunha and F. Novaes, Kerr scattering coefficients via isomonodromy, JHEP 11 (2015) 144 [arXiv: 1506.06588] [INSPIRE].

[54] R.M. Wald and A. Zoupas, A general definition of 'conserved quantities' in general relativity and other theories of gravity, Phys. Rev. D 61 (2000) 084027 [gr-qc/9911095] [INSPIRE].

[55] G. Compere, S. Detournay and M. Romo, Supersymmetric Gödel and warped black holes in string theory, Phys. Rev. D 78 (2008) 104030 [arXiv:0808.1912] [INSPIRE].

[56] E. Tonni, Warped black holes in 3D general massive gravity, JHEP 08 (2010) 070 [arXiv: 1006.3489] [INSPIRE].

[57] S. Detournay and M. Guica, Stringy Schrödinger truncations, JHEP 08 (2013) 121 [arXiv: 1212.6792] [INSPIRE].

[58] G. Compère, W. Song and A. Strominger, New boundary conditions for AdS $S_{3}$, JHEP 05 (2013) 152 [arXiv: 1303.2662] [INSPIRE].

[59] S. Carlip, The statistical mechanics of the $(2+1)$-dimensional black hole, Phys. Rev. D 51 (1995) 632 [gr-qc/9409052] [INSPIRE]. 
[60] S. Carlip, Black hole entropy from conformal field theory in any dimension, Phys. Rev. Lett. 82 (1999) 2828 [hep-th/9812013] [INSPIRE].

[61] S. Carlip, Effective conformal descriptions of black hole entropy, Entropy 13 (2011) 1355 [arXiv:1107.2678] [INSPIRE].

[62] S. Carlip, Effective conformal descriptions of black hole entropy: a review, AIP Conf. Proc. 1483 (2012) 54 [arXiv: 1207.1488] [INSPIRE].

[63] S. Carlip, Black hole entropy from Bondi-Metzner-Sachs symmetry at the horizon, Phys. Rev. Lett. 120 (2018) 101301 [arXiv: 1702.04439] [INSPIRE].

[64] L. Donnay, G. Giribet, H.A. Gonzalez and M. Pino, Supertranslations and Superrotations at the black hole horizon, Phys. Rev. Lett. 116 (2016) 091101 [arXiv:1511.08687] [InSPIRE].

[65] H. Afshar et al., Soft Heisenberg hair on black holes in three dimensions, Phys. Rev. D 93 (2016) 101503 [arXiv:1603.04824] [INSPIRE].

[66] L. Donnay, G. Giribet, H.A. González and M. Pino, Extended symmetries at the black hole horizon, JHEP 09 (2016) 100 [arXiv:1607.05703] [INSPIRE].

[67] H. Afshar et al., Soft hairy horizons in three spacetime dimensions, Phys. Rev. D 95 (2017) 106005 [arXiv: 1611.09783] [INSPIRE].

[68] D. Grumiller, P. Hacker and W. Merbis, Soft hairy warped black hole entropy, JHEP 02 (2018) 010 [arXiv: 1711.07975] [INSPIRE].

[69] H. Gonzalez, D. Grumiller, W. Merbis and R. Wutte, New entropy formula for Kerr black holes, EPJ Web Conf. 168 (2018) 01009 [arXiv: 1709.09667] [INSPIRE].

[70] L. Donnay and G. Giribet, Cosmological horizons, Noether charges and entropy, Class. Quant. Grav. 36 (2019) 165005 [arXiv: 1903.09271] [INSPIRE].

[71] L. Donnay and C. Marteau, Carrollian physics at the black hole horizon, Class. Quant. Grav. 36 (2019) 165002 [arXiv: 1903.09654] [INSPIRE].

[72] D. Grumiller et al., Spacetime structure near generic horizons and soft hair, arXiv:1908.09833 [INSPIRE].

[73] C. Troessaert, Enhanced asymptotic symmetry algebra of $A d S_{3}$, JHEP 08 (2013) 044 [arXiv: 1303.3296] [INSPIRE].

[74] S.G. Avery, R.R. Poojary and N.V. Suryanarayana, An sl $(2, \mathbb{R})$ current algebra from $A d S_{3}$ gravity, JHEP 01 (2014) 144 [arXiv: 1304.4252] [INSPIRE].

[75] D. Grumiller and M. Riegler, Most general AdS $S_{3}$ boundary conditions, JHEP 10 (2016) 023 [arXiv: 1608.01308] [INSPIRE].

[76] A. Pérez, D. Tempo and R. Troncoso, Boundary conditions for General Relativity on $A d S_{3}$ and the KdV hierarchy, JHEP 06 (2016) 103 [arXiv: 1605.04490] [INSPIRE].

[77] T. Hartman, C.A. Keller and B. Stoica, Universal spectrum of $2 d$ conformal field theory in the large c limit, JHEP 09 (2014) 118 [arXiv:1405.5137] [INSPIRE]. 